http://jmscr.igmpublication.org/home/ ISSN (e)-2347-176x ISSN (p) 2455-0450 crossref DOI: https://dx.doi.org/10.18535/jmscr/v9i9.09

\author{
Journal Of Medical Science And Clinical Research

\title{
Radiation Therapy in Rosai Dorfman Disease Involving Long Bone- A Case Report
}

Authors

\author{
Dr Himanshi Jain ${ }^{1}$, Dr Maitrik Mehta ${ }^{2}$, Dr U. Suryanarayan ${ }^{3}$, Dr Ankita Parikh ${ }^{4}$, \\ Dr Dinesh Anand ${ }^{5}$, Dr Krishna Ratanchandani ${ }^{6}$, Dr Nikhil Bathija ${ }^{7}$, Dr Viraj Modi ${ }^{8}$, \\ Dr Siddharth Malukar', Dr Dhara Patel ${ }^{10}$ \\ ${ }^{1,5,6,7,8,9,10}$ Resident Doctor, ${ }^{2}$ Associate Professor, ${ }^{3} \mathrm{Head}$ of Department, ${ }^{4}$ Professor
}

\section{Introduction}

Rosai Dorfman disease, a rare, benign, proliferative, histiocytic disorder is also known as sinus histiocytosis with massive lymphadenopathy. Patients with this disease usually present with fever leukocytosis and painless cervical lymphadenopathy. It can also present at extra-nodal sites, commonly skin, soft tissue, central nervous system, and rarely bone.

There are no established treatment guidelines in this disease and there are very few reports discussing radiation therapy in Rosai Dorfman disease, although various modalities can be used for the treatment including surgery and chemotherapy also.

We present a case of 27 year old male patient with Rosai Dorfman disease of right knee treated at our institute and approach to its management. Patient is on monthly follow up since 6 months and is symptom free.

\section{Case Report}

A 17-year old male was diagnosed with Pre-B cell Acute Lymphoblastic Leukemia in 2010. Patient was on MCP-841 protocol, received prophylactic cranial irradiation in April 2010. He completed chemotherapy in May 2012. He was kept on follow-up since then and had no relapse later.

He then presented with complain of swelling in right knee in 2019, which was associated with moderate pain. A MRI of the right knee joint was done which was suggestive of an altered signal intensity lesion involving meta-epiphyseal region of the distal medial femoral condyle. FNAC was attempted from the lesion and no opinion was possible from that.

Patient then was loss to follow-up for 1 year. In January 2021, the same patient presented with complain of swelling in bilateral knee joint associated with pain in the right knee. On examination there was a mass present on the right knee joint of approximately $9 * 8 \mathrm{~cm}$ which was hard on touch and non-tender, with swelling over upper part of right tibia .A MRI of bilateral knee joint was done which showed multiple lesion involving lower meta and diaphysis of both femur and upper diaphysis of right tibia and fibula. An exophytic lesion $(7.9 * 5.3 \mathrm{~cm})$ involving lower meta and diaphysis of right femur extending posteriorly in popliteal fossa. 
Biopsy from the distal femur showed possibility of Rosai Dorfman disease. Immunohistochemistry was done to confirm the diagnosis. (table 1)

\begin{tabular}{|c|c|}
\hline MARKER & STATUS \\
\hline S100 & Positive \\
\hline LCA & Positive \\
\hline CD68 & Positive \\
\hline Tdt & Negative \\
\hline
\end{tabular}

On discussion with the pathologist an another marker CD1a was added to rule out Langerhans cell histiocytosis. The marker CD1a was negative confirming Rosai Dorfman disease.

Patient underwent a pet- ct scan diagnostic scan which showed a $6.6 * 4.5 * 9.5 \mathrm{~cm}$ lesion in both lobe of liver (suv-17.50). Metabolically active lesion in right lower end of femur- both metaphysis and epiphysis of size- $8.4 * 5.4 * 8.2 \mathrm{~cm}$ with bone marrow involvement. Another lesion was present in the left tibia of size- $6.1 * 7.6 * 8.7$ $\mathrm{cm}$ involving medial end of shaft of tibia both metaphysis and diaphysis (suv-10.58) with bone marrow involvement. A usg guided biopsy from the lesion in the right lobe of liver was done which showed diffuse fragments of liver tissue diffusely infiltrated by inflammatory cells with spindly cells with moderate cytoplasm. Immunohistochemistry favoured histocytic lineage tumor. How-ever since s100 was negative, Rosai Dorfman disease and Langerhans cell histiocytosis were ruled out. Tdt was also negative ruling out leukemic infiltration. After a multidisciplinary discussion and review of literature, patient was treated with external radiotherapy by conventional 2-D technique, 3000 cGy in 15 fractions, 5 days a week to the right knee using cobalt-60 machine- AP-PA technique. Patient developed grade 1 skin reaction after completion of radiotherapy. The patient was relieved from the complain of pain after receiving $10 \mathrm{gy}$. How-ever after reviewing of literature the dose was escalated to $30 \mathrm{gy}$. At the end of radiotherapy treatment there was decrease in the swelling of the local part. Patient is now on inj. zolendronic acid.
On follow up after 1 month post radiotherapy the patient had no symptoms for the treated site (ECOG-0). The patient is on monthly follow up at present and has no complains.

\section{Discussion}

Rosai Dorfman Disease was initially described by Destombes in 1965, and was characterized by Rosai and Dorfman in 1969 as a clinicopathological disorder. $^{(1,2)}$

Bone involvement occurs in less than $10 \%$ of cases.

Rosai Dorfman Disease is a disease of benign histiocytes which infiltrates lymph nodes or extranodal tissues. The cells exhibit emperipolesis (presence of an intact cell within the cytoplasm of other cell), the non-destructive phagocytosis of lymphocytes or erythrocytes, which is the hallmark of the disease and essential for diagnosis. The etiology of RDD is yet unknown. There are some evidence suggesting that immune dysfunction and viral infections, such as human herpesvirus (HHV), parvovirus B19, and EpsteinBarr virus (EBV) may play a role in the pathogenesis. ${ }^{(3,4,5)}$

Typically, RDD manifests in childhood and early adulthood, with the majority of cases reported in the second and third decades of life. Majority of the patients present with symptoms of fever and non painful cervical lymphadenopathy similar to that of lymphoma, associated with night sweats and weight loss. The workup includes detailed history and physical examination. Contrast CT scans and magnetic resonance imaging as well as PET-CT can be done to look for distant disease. Bone marrow biopsy is usually done because primary bone marrow disorders are included in the differential diagnosis of RDD. Screening for EBV, cytomegalovirus, HHV-6, HHV-8, and HIV maybe done. Also the workup should include complete blood counts, liver and kidney function tests, rheumatoid factor, an antinuclear antibody test, immunoglobulin levels, and an erythrocyte sedimentation rate (ESR). Biopsy is useful for diagnosis and which is further confirmed by 
immunohistochemistry. The differential diagnosis of RDD is broad and described in the table (2).

\begin{tabular}{|c|c|}
\hline NON MALIGNANT & MALIGNANT \\
\hline TUBERCULOSIS & HODGKIN LYMPHOMA \\
\hline WEGENER & NON HODGKIN \\
GRANULOMATOSIS & LYMPHOMA \\
\hline SARCOIDOSIS & MELONAMA \\
\hline GAUCHER DISEASE & LANGERHANS CELL \\
& SARCOMA \\
\hline LANGERHANS CELL & \\
HISTIOCYTOSIS & \\
\hline
\end{tabular}

Extra-nodal involvement by RDD was initially considered uncommon, however some reports suggest that it may be present in up to $40 \%$ of cases. In majority of the cases the involved extranodal sites include the skin, CNS, orbit and eyelid, upper respiratory tract, and the gastrointestinal tract.

RDD histiocytes will be positive for immunohistochemical stains CD68 (KP-1), CD163, and S100 and are typically negative for CD1a. Because RDD is a non-malignant histiocytic disorder, treatment for the disease is advised only in patients who are symptomatic or have vital organ or system involvement (i.e., CNS). In RDD, $20 \%$ of cases may show spontaneous regression without any therapy. Relapsing and remitting RDD without treatment may occur in another $70 \%$ of patients, complicating the timing of when to use therapy.

Less than $5 \%$ of reported cases of RDD may include skeletal involvement, it is difficult to establish the best treatment for these patients. ${ }^{(6)}$. According to a study, only $10 \%$ of the total rdd in bone received radiotherapy ${ }^{(7)}$. While many authors have argued that the disease is generally indolent and tends to wax and wane with regard to symptoms, others have argued that without treatment $70-80 \%$ of patients experience persistent disease with at least intermittent symptoms. Most authors agree that surgical removal of the lesion is a mainstay of treatment. A more aggressive surgical approach, namely en block resection of the distal femur may have been considered in this patient. With this approach the risk of local recurrence would have been negligible, but the patient would have to undergo reconstruction of the skeletal defect along with a distal femoral replacement knee arthroplasty. Since the disease is benign, resection was not favoured.

The role of radiotherapy as a treatment modality for RDD is yet inconclusive. Recommendations for dose of external beam radiotherapy vary broadly. Some of the authors recommend doses between 20 to $30 \mathrm{~Gy}^{(6,8,9)}$ and other authors reporting treatment doses higher than $50 \mathrm{~Gy}^{(10,11)}$. Since in our patient the lesion involved distal femur near the joint, dose was limited to 30Gy. Some authors also state that chemotherapy is of limited benefit in the treatment of these patients ${ }^{(8)}$.Chemotherapeutic agents such as antimetabolites, alkylators, and vinca alkaloids can be used, but mostly with limited benefit. Hence after multidisciplinary discussion, and after giving and all the options to the patient and caretaker, radiotherapy as the treatment of choice in this patient was decided. However, due to the rarity of the disease, it is unlikely that there will ever be definitive clinical guidelines for the treatment of RDD in bone. While the optimal dose regimen for radiotherapy has not yet been established, symptomatic patients do appear to benefit from external beam radiotherapy for extranodal RDD even at doses as low as $10 \mathrm{~Gy}$.

\section{Conclusion}

In conclusion, we can say that rdd being a rare and mostly self-limiting benign disease with no clear treatment protocol has been established. Majority of the patients are asymptomatic and in patients with symptomatic disease, radiation therapy is a reasonable option, providing adequate symptom relief.

\section{References}

1. Rosai J, Dorfman RF. Sinus histiocytosis with massive lymphadenopathy: a pseudolymphomatous benign disorder. Analysis of 34 cases. Cancer 1972;30:1174- 88.

2. Rosai J, Dorfman RF. Sinus histiocytosis with massive lymphadenopathy. A newly 
recognized benign clinicopathological entity. Arch Pathol1969;87:63-70.

3. Foucar E, Rosai J, Dorfman R. Sinus histiocytosis with massive lymphadenopathy (Rosai-Dorfman disease): review of the entity. Semin Diagn Pathol. 1990;7(1):19-73.

4. Mehraein Y, Wagner M, Remberger K, et al. Parvovirus B19 detected in RosaiDorfman disease in nodal and extranodal manifestations. J Clin Pathol. 2006;59 (12):1320-1326.

5. Noguchi S, Yatera K, Shimajiri S, et al. Intrathoracic Rosai-Dorfman disease with spontaneous remission: a clinical report and a review of the literature. Tohoku J Exp Med. 2012;227(3):231-325.

6. Walker PD, Rosai J, Dorfman RF. The osseous manifestations of sinus histiocytosis with massive lymphadenopathy. Am J Clin Pathol 1981;75:131-9

7. Bone Involvement in Rosai-Dorfman Disease (RDD): a Case Report and Systematic Literature Review Birgit A. Mosheimer, Bastian Oppl, Shahin Zandieh, Michael Fillitz, Felix Keil, Klaus Klaushofer, Günter Weiss, Jochen Zwerina Curr Rheumatol Rep. 2017; 19(5): 29. Published online 2017 Apr 11. doi: 10.1007/s11926-017-0656-6

8. Toguri d, Louvie AV RizkallaK,et al. Radiotherapy for steroid-resistant laryngeal Rosai-Dorfman disease. Curr Oncol 2011;18:e158-62.

9. Rodriguez-Galindo C, Helton KJ, Sanchez $\mathrm{ND}$, et al. Extranodal Rosai-Dorfman disease in children. J Ped Hematol Oncol 2004;26:19-24.

10. Kelly WF, Bradey N, Scoones D. Rosai Dorfman disease presenting as a pituitary tumour. Clin Endocrinol 1999;50:133-7.
11. Childs HA 3rd, Kim RY. Radiation response of Rosai-Dorfman disease presenting with involvement of the orbits. Am J Clin Oncol 1999;22:526-8. 